\section{EMBRYRIDDLE}

Aeronautical University

SCHOLARLY COMMONS
Journal of Aviation/Aerospace

Education \& Research

Volume 29

Number 2 JAAER 2020

Article 4

2020

\title{
Dynamic Delegated Corridors and 4D Required Navigation Performance for Urban Air Mobility (UAM) Airspace Integration
}

Trong Van Nguyen

Embry-Riddle Aeronautical University, nguyet58@my.erau.edu

Follow this and additional works at: https://commons.erau.edu/jaaer

Part of the Aeronautical Vehicles Commons, Aviation Safety and Security Commons, Management and Operations Commons, Multi-Vehicle Systems and Air Traffic Control Commons, Navigation, Guidance, Control and Dynamics Commons, and the Risk Analysis Commons

\section{Scholarly Commons Citation}

Nguyen, T. V. (2020). Dynamic Delegated Corridors and 4D Required Navigation Performance for Urban Air Mobility (UAM) Airspace Integration. Journal of Aviation/Aerospace Education \& Research, 29(2). https://doi.org/10.15394/jaaer.2020.1828

This Article is brought to you for free and open access by the Journals at Scholarly Commons. It has been accepted for inclusion in Journal of Aviation/Aerospace Education \& Research by an authorized administrator of Scholarly Commons. For more information, please contact commons@erau.edu. 


\section{Introduction}

Increased traffic congestion on urban road networks has impacted the travel time for commuters in highly populated urban centers. Thus, there is a need within the industry to develop other means of transportation to mitigate this problem. Urban Air Mobility (UAM) may offer a solution. UAM is recognized as a system that transports the passenger and air cargo from any location to any destination within an urban area to potentially overcome the increasing road congestion of automobile urban surface transportation in crowded environments (Reiche, McGillen, Siegel, \& Brody, 2019). UAM provides an alternative mode of transportation to enhance mobility instead of commuters' private vehicles to reduce the time spent on daily travel (National Aeronautics and Space Administration [NASA], 2019).

The characterization of UAM aircraft using electric vertical takeoff and landing (eVTOL) vehicles have been developed as a viable mode of future autonomous air taxi services and could be a reality by 2030 (NASA, 2018). UAM is described as "a set of vehicles and operational concepts that proposes to develop short-range, point-to-point transportation systems in metropolitan areas using vertical takeoff and landing (VTOL) or short takeoff and landing (STOL)" (Vascik, Hansman, \& Dunn, 2018, p. 1). UAM aircraft include unmanned aircraft systems (UAS), air-taxi passenger-carrying aircraft, and an aircraft with a pilot onboard, remote pilot on the ground, and progressively self-flying operations (Reiche et al., 2019).

Since the predicted demand for UAM aircraft to be integrated into the existing airspace system in the next ten years and beyond is at high volume, the aviation industry needs to address safety as one of the challenges under today's constructs to meet the safety requirements for provision to passenger carrying UAM. UAM may have potential utilization in many areas, such as non-scheduled urban air taxi (on-demand) flights, personal traveling, air emergency services, 
and policing services (Reiche et al., 2019). The aviation industry needs to address the technological, physical, functional, and integrational challenges of system-of-systems depending on each other to a large degree to make UAM viable (NASA, 2018). Many of the most critical problems to UAM are the capacity of the existing air traffic management system, the accessibility of take-off and landing areas and charging systems on the ground, regulations and certifications, adverse weather conditions, pilot shortage, and public acceptance of aircraft noise (Vascik \& Hansman, 2018). These challenges could hinder implementation and limit the sustainable growth of UAM systems.

To tackle these challenges, many organizations in the aviation industry, academia, and government have conducted extensive studies on the issue of UAM vehicle development and technology. The National Aeronautics and Space Administration (NASA) has carried out numerous ongoing research projects that substantially support UAM. These continuing projects include "UAS Traffic Management (UTM) \& UAS Integration in the NAS (UAS-NAS), Flight Demonstrations and Capabilities (FDC), X-57, Air Traffic Management - Exploration (ATM-X), System Wide Safety (SWS), Transformative Tools \& Technologies (TTT), Revolutionary Vertical Lift Technologies (RVLT), and Advanced Air Mobility (AAM)” (NASA, 2019, p. 11). UAS are currently operating at low altitudes (e.g., below 400 feet). UAS have used the Unmanned Aircraft Systems (UAS) Traffic Management (UTM) services, which provides a set of traffic management services via a federated group of UTM Service Suppliers (USS), similar to traditional Air Traffic Control (ATC) services provided to IFR and VFR aircraft (Lascara et al., 2019). According to Ghayouraneh, El-Ghazaly, and Rankin (2018), UAM operations are expected to occur above 400 feet (between 1,000-3,000 feet). Even when transitioning from higher airspace to lower airspace (below 400 feet), UAM aircraft may not be able to leverage 
some existing UTM services because there is a need to modify the service reliability designed for small UAS operations to meet the safety requirements for provision to passenger carrying UAM (Lascara et al., 2019). UAM aircraft may create more complexity, more delays, and an increased hindrance to the current airspace system's existing facilities and resources. Therefore, it is essential to develop specialized airspace sectors and trajectories to allow UAM aircraft to operate safely and efficiently in specific corridors, which are separated from other airspace users at low altitudes in controlled airspace. This research aims to assess the combination of the Dynamic Delegated Corridors (DDCs) and full four-dimensional (4D) Required Navigation Performance (RNP) trajectories to enable a safe and efficient airspace integration of UAM aircraft into the terminal control airspace at low altitudes.

\section{Research Questions}

The following research questions and hypotheses will be addressed in this study:

RQ1: What impacts does the DDC have on airspace managers and air traffic managers controlling that airspace?

RQ2: What impacts does the 4D RNP trajectory have on airspace managers and air traffic managers controlling that airspace?

RQ3: What impacts does the interaction between DDC and the 4D RNP trajectory have on airspace managers and air traffic managers controlling that airspace?

\section{Statement of the Problem}

The existing airspace structure was designed and allocated for conventional aircraft, which have different performance and separation requirements from the UAM aircraft (Vascik \& Hansman, 2018). As opposed to manned aircraft, the UAM aircraft have slower airspeed, increased manoeuvrability, vulnerability to wake turbulence, as well as command and control 
differences. The projected UAM operations and high demand for UAM airspace integration into the existing airspace system may result in more workload for ATC and exceed the capacity of current ATC system resources (Vascik, Hansman, \& Dunn, 2018). For this reason, during the peak hour with high traffic congestion, the operations of UAM aircraft may be delayed, restricted, or even prohibited in specific airspace due to the limited ATC workload (Vascik, Hansman, \& Dunn, 2018; Verma, Keeler, Edwards, \& Dulchinos, 2019). Thus, the need for developing new airspace management constructs and operational procedures for the current airspace system is essential to reduce airspace manager and air traffic manager workload to enable the accommodation of projected UAM operations, thereby increase the viability of UAM airspace integration.

\section{Literature Review}

The operations of UAM aircraft for commercial purposes have existed in the United States since the 1940s. Helicopters were used by Los Angeles Airways to transport people and mail between many locations in Los Angeles from 1947 to 1971 (Thipphavong et al., 2018). UAM concepts have been proposed continuously since the 1960s. After Uber company announced its intention to build an "air taxi" to supplement transportation on the ground in 2016, many concepts and proposals for the UAM role were motivated by this announcement (Kadhiresan \& Duffy, 2019). Several different aircraft configurations have been considered, including multirotors, tiltrotors, and conventional helicopters (Kadhiresan \& Duffy, 2019; Thipphavong et al., 2018). However, these systems were not implemented because of many associated challenges, such as commercial viability concerns, noise acceptance from community, and air traffic control capacity (Thipphavong et al., 2018; Vascik, Hansman, \& Dunn, 2018). According to Moore (2010), the On-Demand Aviation (ODA) system was developed by NASA. 
ODA was designed based on small electric aircraft and advanced autonomy to allow vehicles to operate within and around a city with a radius of 165 miles.

NASA developed the initial UTM Concept of Operations (ConOps), which is concentrated on safely enabling large-scale sUAS operations in low altitude uncontrolled airspaces (Class G airspace) (Kopardekar et al., 2016). UTM ConOps combines the construct of airspace, flight rules, method of operation, automatic systems from the ground, with the abilities of the vehicle to enable sUAS to operate safely in the current airspace system (Kopardekar et al., 2016). With a heightened focus on the combinations of concepts, procedures, and technologies, Mueller, Kopardekar, and Goodrich (2017) recommended that the UAM operation can leverage the concepts and technologies developed for the UTM system. The concepts and technologies developed for sUAS at low altitudes can be extended to higher levels to support for integration, evaluation, and deployment of UAM concepts at low, medium, and high density airspace. Mueller et al. (2017) also indicated that research to enable airspace integration of UAM aircraft mostly depends on the level of automation of aircraft, so UAM aircraft can operate relatively independently and will not receive ATC services from the current air traffic control system. Therefore, UAM flights are not dependent on ATC capacity limits.

In the "Concept for Urban Airspace Integration DLR U-Space Blueprint" paper, the DLR (German Aerospace Center) suggests a system to manage airspace at high-density traffic for future Unmanned Traffic Management (UTM) or U-space (Geister \& Korn, 2018). This concept concentrates on integrating novel airspace users, such as UAS and Urban Air Taxis, into uncontrolled, Class G airspace. Depending upon the individual performance parameters of aircraft, each aircraft is modeled by an ellipsoid, which is the airspace partitioned into cells of the same requirements on airspace usage (Geister \& Korn, 2018). If the aircraft has an overall low 
performance in relation to navigation, communication, and the detecting capabilities relative to other aircraft (cooperatively and uncooperatively), it will be assigned to the larger resulting safety ellipsoid (Geister \& Korn, 2018), as illustrated in Figure 1.

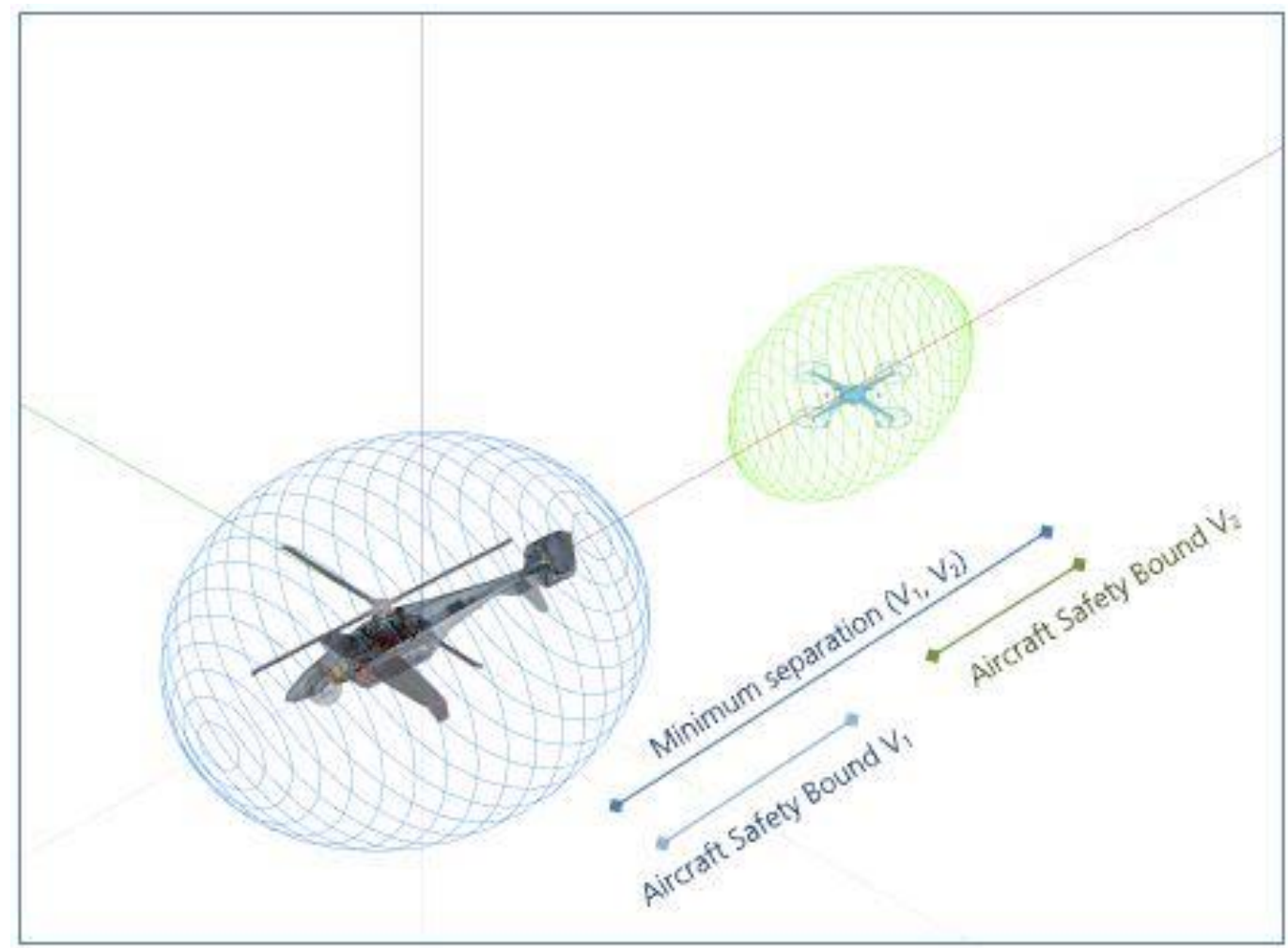

Figure 1. Relative separation distances considering different aircraft types (Geister \& Korn, 2018).

The strength of the DLR Blueprint concept is that it allows UAS with both high and low technical equipment levels to have equal accessibility within the airspace. The proposed DLRU-space concept splits the airspace into individual segments of the same characteristics. Since the airspace segments are reliant on their capacity on the separation requirements of the airspace users operating in that, aircraft with different specific equipment and capability may have different separation requirements and, in this way, also dynamically influence the capacity of an 
airspace segment. This DLR Blueprint concept results in airspace management, which allows more flexibility for aircraft operating at low density but little freedom at high density due to separation requirement constraints (Geister \& Korn, 2018).

Several studies on UAM are focused on identifying operational capabilities and constraints for urban air mobility (Vascik \& Hansman, 2018; Vascik, Hansman, \& Dunn, 2018). One study conducted by Vascik and Hansman (2018) identified three critical operational constraints, which are noise, infrastructure, and ability of the current air traffic control system to grow larger that may restrict the employment or advancement of UAM systems. Mainly, the current ATC workload limitations and the required minimum separation standard applied to conventional aircraft are difficult to employ for UAM aircraft. Thipphavong et al. (2018) carried out a high-level framework "Urban Air Mobility Airspace Integration Concepts and Considerations" project for integrating UAM aircraft at a low level of risk, low tempo, and low density. Thipphavong et al. (2018) investigated the challenges associated with integration. These challenges include planning for the UAM mission, separating UAM from other aircraft, terrain, and obstacles, managing contingency, maintaining a balance between demand and capacity, and managing traffic flow (Thipphavong et al., 2018).

Vascik, Hansman, and Dunn (2018) conducted three exploratory case studies of three cities: Los Angeles, Boston, and Dallas. When reviewing the ConOps for 32 reference missions planned to provide UAM services in Los Angeles, Boston, and Dallas, Vascik, Hansman, and Dunn (2018) stated that autonomous aircraft interactions with ATC would have a significant impact on other airspace users in controlled airspace and pointed out the 19 operational challenges that may hinder or influence the near or far term UAM advancement, application, or operation. From the 19 identified constraints, Vascik et al. (2018) determined eight overarching 
UAM operational limitations, in which five potential UAM constraints directly correlated with operational issues that the air carrier operations conducted in a helicopter previously identified. The continuation of these restrictions signifies that projected operations of UAM may further be impeded even though new technologies and business models are available (Vascik, Hansman, \& Dunn, 2018).

Verma et al. (2019) used the airspace of the Dallas Fort Worth area, consisting of Class B and D airspace, to explore potential routes and procedures to allow UAM aircraft to integrate into the airspace. The results show that the existing routes flown by helicopter and methods of communication may help to manage UAM operations in the near-term but may not meet the requirements for the middle-term and long-term. Verma et al. (2019) also pointed out that unlike sUAS operation, which does not require take-off and landing areas (TOLAs), UAM flight requires ground infrastructure such as a network of vertiports and charging systems. UAM operations also necessitate scheduled and separation services, the same as what is currently provided to manned aircraft by ATC.

Lascara et al. (2019) conducted a study on the subject of "Urban Air Mobility Airspace Integration Concepts" at Massachusetts Institute of Technology Research and Engineering Corporation (MITRE) and proposed four main concept components required to enable UAM airspace integration: augmented visual flight rules, Dynamic Delegated Corridors (DDCs), automated decision support services, and performance-based operations. Figure 2 shows an example flight trajectory of an automated UAM aircraft flying through different airspace classes with dynamic delegated corridors (Lascara et al., 2019). 


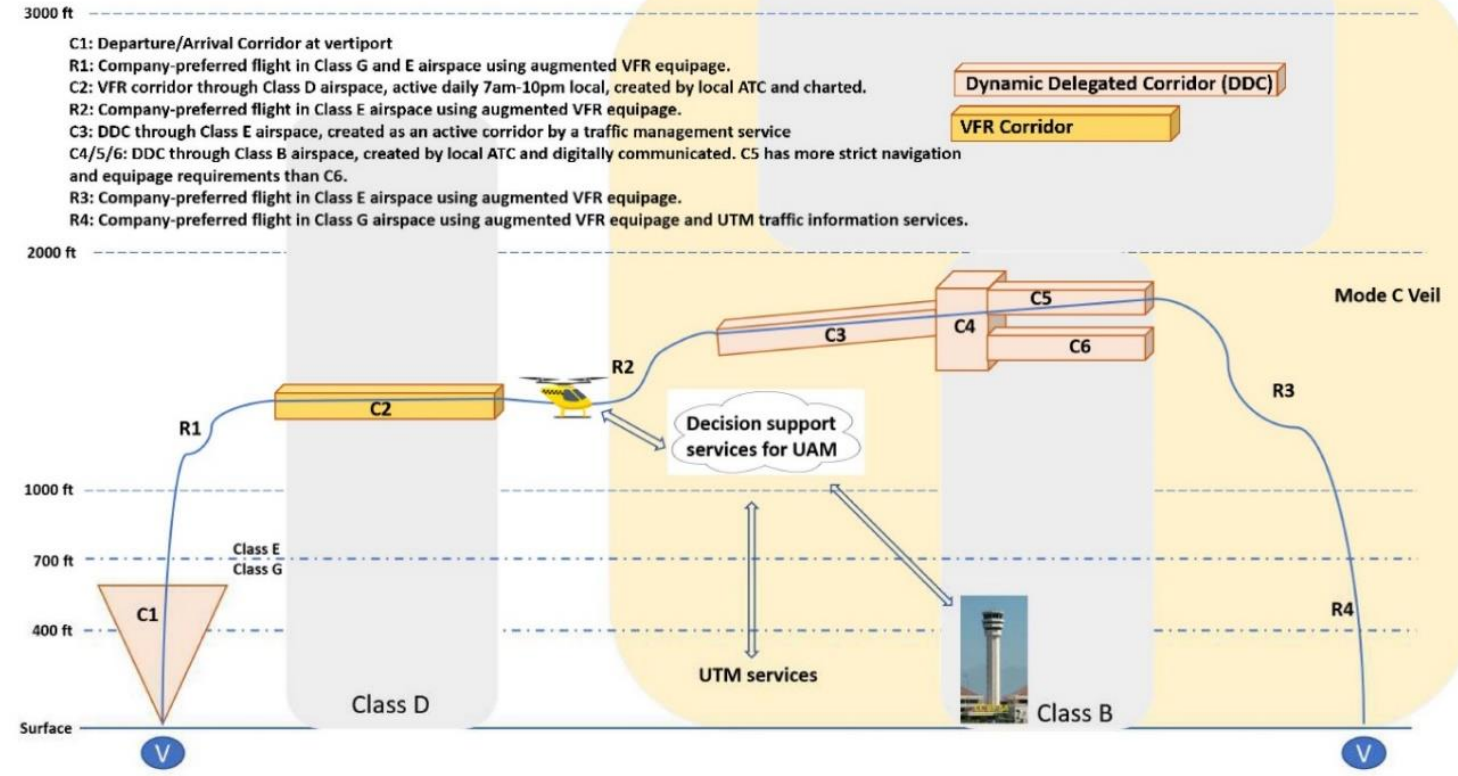

Figure 2. Notional Depiction of Airspace Integration Concepts (Lascara et al., 2019).

- C1) As shown in Figure 2, UAM aircraft follow the departure/arrival corridor (DDC) at vertiport to establish a procedural separation between unmanned aircraft.

- R1) After climbing and passing altitude 400 feet, UAM aircraft leave departure DDC, enter Class $\mathrm{G}$ airspace, and climb to 700 feet. Then UAM aircraft continue to climb to 1,500 feet in Class E airspace.

- C2) After reaching 1,500 feet and leaving R1, UAM aircraft can either enter Class D airspace or fly through the VFR corridor if UAM aircraft is equipped with augmented VFR technology.

- R2) After exiting VFR corridor C2 and re-entering Class E airspace, UAM aircraft follow R2 company-preferred route flying towards its destination.

- C3) UAM aircraft enter VFR corridor (DDC) C3 in a portion of Class E airspace. 
- C4/5/6) After leaving DDC C3, UAM aircraft can either navigate around Class B airspace or fly through DDC C4, then DDC C5 or DDC C6. Corridor C5 requires higher navigation precision performance and provides a shorter flight trajectory than Corridor C6.

- R3) UAM aircraft equipped with augmented VFR descend on the R3 route towards its destination.

- R4) After changing from Class E to Class G airspace, UAM aircraft make the final approach and land at vertiport, which has no DDCs. UAM aircraft employ augmented VFR and UTM traffic information services to avoid and maintain separation with other UAM traffic and other small UAS in the landing site.

However, in this study, Lascara et al. (2019) did not specify clearly what navigation performance requirements (i.e., the accuracy of containment) will be mandatory for UAM aircraft to operate in DDCs. Thus, the challenge to address is how to ensure the operation of UAM aircraft along a precise flight path of DDCs with a high accuracy level and the capability to determine aircraft position with both precision and integrity.

\section{Proposed DDCs and 4D RNP Trajectory Concept}

Based on the identified operational challenges and functional characteristics of UAM aircraft, this study will assess the combination of DDCs and 4D RNP trajectory to enable UAM airspace integration. DDCs will allow UAM aircraft to operate in the corridors, which are procedurally separated from conventional aircraft. 4D RNP trajectory is the extension of the existing International Civil Aviation Organization (ICAO) RNP and includes the required navigation performance for the vertical and along-track dimension with consideration for a time as well (Dautermann, 2016). 4D RNP trajectories, which will be built along the DDCs, provide latitude, longitude, altitude, and time information to the air traffic control system. 4D RNP 
trajectories enhance the precision navigation capability, which will allow for the reduced lateral or longitudinal separation buffers controllers add to account for uncertainty (Federal Aviation Administration [FAA], 2016). Thus, 4D RNP trajectories will enable UAM aircraft to stay within DDCs with a high level of accuracy.

Recently, NASA developed a quantitative framework to adopt RNP to UAS applications based on operational risk (Jung et al., 2016). The existing standard approach RNP 0.3 (1823 $\mathrm{ft} / 556 \mathrm{~m})$ and minimum RNP $0.1(607 \mathrm{ft} / 185 \mathrm{~m})$ defined by ICAO for manned aircraft cannot be directly applied to UAS or UAM since separation minima for unmanned aircraft do not currently exist. These standards are too large for UAM and UAS platforms in view of their intended applications (Bijjahalli, Sabatini, \& Gardi, 2019). The Fédération Aéronautique Internationale (FAI) specified the preliminary limits for navigation performance. The preliminary standard horizontal Performance Based Navigation (PBN) specification for UAS is set at $15 \mathrm{~m}$, and the preliminary precision horizontal specification is set at $3 \mathrm{~m}$. The vertical PBN can apply nominal values of $30 \mathrm{~m}$ (standard) and $15 \mathrm{~m}$ (precision) (Bijjahalli et al., 2019). Currently, there are no standardized minimum performance requirements for UAM navigation systems. For that reason, a limiting threshold value approximately equal to one-third of an average urban street width (10 $\mathrm{m})$ is set to compute the system availability, which lies between the standard and precision PBN boundaries mentioned above (Bijjahalli et al., 2019). Figure 3 illustrates the DDCs and 4D RNP trajectory concepts. 


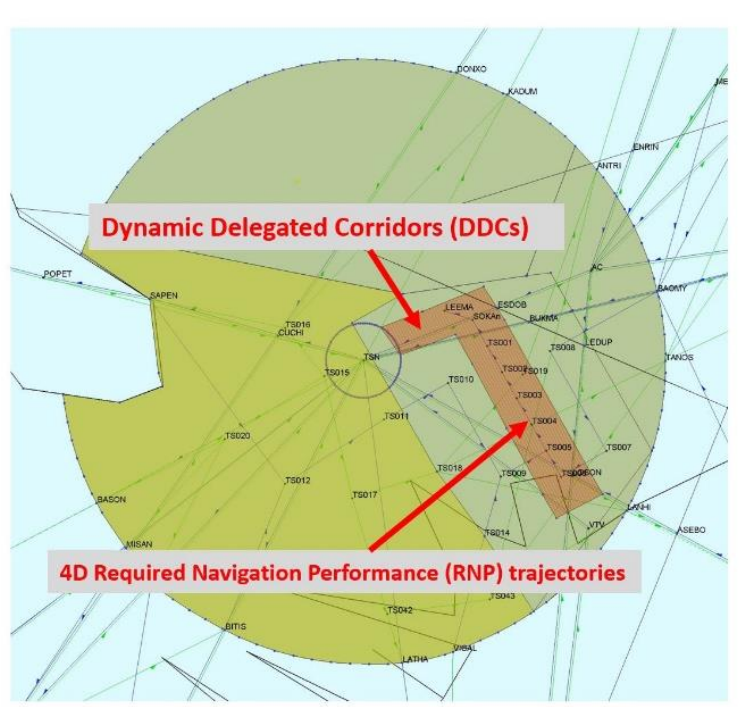

Dynamic Delegated Corridors (DDCs) \& 4D RNP Trajectory in plan view

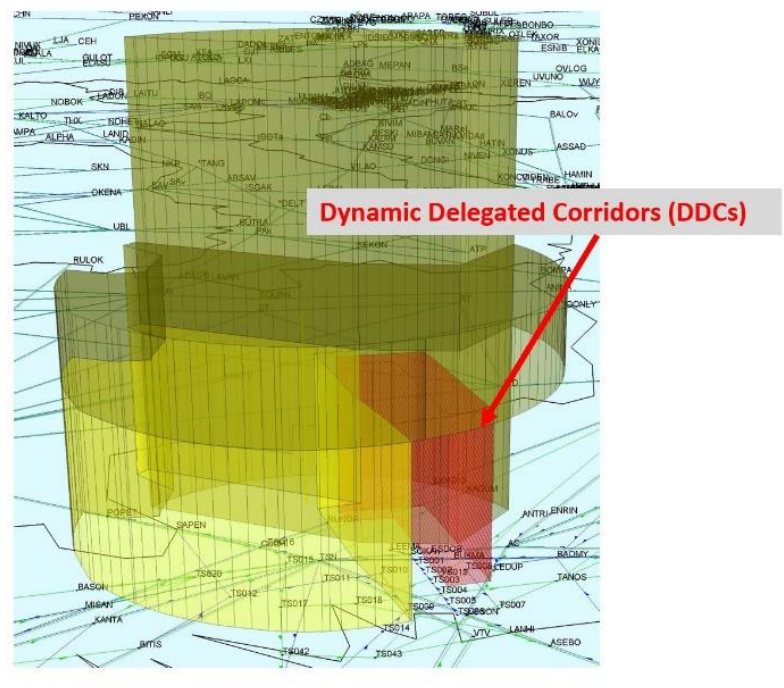

Dynamic Delegated Corridors (DDCs)

\& 4D RNP Trajectory in side view

Figure 3. DDCs and 4D RNP trajectory concepts. Created by author.

\section{Discussion and Conclusion}

The purpose of the present study was to analyze and synthesize existing literature about UAM airspace integration concepts that have been developed across the aviation industry in the United States and other countries. Then the introduction of the Dynamic Delegated Corridors (DDCs) and full four-dimensional (4D) Required Navigation Performance (RNP) trajectories are proposed to enable UAM aircraft to integrate into the existing airspace system.

The NASA UTM ConOps developed for sUAS at low altitudes can be used to support the integration, evaluation, and deployment of UAM concepts at low, medium, and high-density airspace by extending to higher levels (Mueller et al., 2017). The DLR (German Aerospace Center) concept for urban airspace integration provides more flexibility for UAS at low density but little independence at high density (Geister \& Korn, 2018). The operational challenges identified by Vascik, Hansman, and Dunn (2018) may have an influence on UAM operation, development, and implementation in the near term or long term. The MITRE's "Urban Air Mobility Airspace Integration Concepts" proposed four main concept components required to 
enable UAM airspace integration: augmented visual flight rules, Dynamic Delegated Corridors (DDCs), automated decision support services, performance-based operations (Lascara et al., 2019). However, in the proposal of MITRE conducted by Lascara et al. (2019), there was no precise specification of what navigation performance requirements that UAM aircraft must meet to operate in DDCs.

The approach taken in the current study is to assess the combination of the DDCs and full four-dimensional 4D RNP trajectories to enable UAM aircraft to integrate into the existing airspace system. The proposed combination of DDCs and 4D RNP in the present study is expected to help reduce the workload of ATC dramatically, which may contribute to the UAM airspace integration and operation at low altitudes in the terminal control airspace together with other airspace users.

The current airspace system, regulations, policies, and structures are not yet well developed to accommodate envisaged UAM operations. However, with the support of digital data communication — and automated decision support tools for ATC, Trajectory Based Operations (TBO), the proposed combination of DDCs and 4D RNP in the current study - may enable air traffic controllers to accommodate the projected demand for UAM operations, so that UAM airspace integration can be feasible by 2030 and beyond.

Nevertheless, future work and research is needed to define the operational requirements UAM aircraft must meet (Thacker, 2018). Also, the aviation industry needs to look for new technologies to enhance navigational precision of UAM aircraft flying in DDCs and provide the ability for UAM aircraft to operate the most optimal trajectory. The improved performances of 4D RNP may help reduce separation, support better flow planning, and enhance the ability to manage the imbalances of demand-capacity of UAM operations (FAA, 2016; Hartmann, 2013). 


\section{References}

Bijjahalli, S., Sabatini, R., \& Gardi, A. (2019). GNSS performance modelling and augmentation for urban air mobility. Sensors, 19(19), 4209. https://doi.org/10.3390/s19194209

Dautermann, T. (2016, Spring). Extending required navigation performance to include time based operations and the vertical dimension. Navigation - Journal of the Institute of Navigation, 63(1), pp. 53-64. https://doi.org/10.1002/navi.124

Federal Aviation Administration (FAA). (2016, June). The future of the NAS. U.S. Department of Transportation. Retrieved from https://www.faa.gov/nextgen/media/futureofthenas.pdf

Geister, D., \& Korn, B. (2018). Density based management concept for urban air traffic. IEEE/AIAA 37th Digital Avionics Systems Conference (DASC), London, England. https://doi.org/10.1109/DASC.2018.8569491

Ghayouraneh, S., El-Ghazaly, S. M., \& Rankin, J. M. (2018). Dynamic addressing for ondemand mobility. AIAA Aviation Forum, 2018 Aviation Technology, Integration, and Operations Conference, Atlanta, GA. https://doi.org/10.2514/6.2018-4152

Hartmann, S. (2013). Information analysis for a future flight deck design in the context of 4D trajectory based operation. Proceedings of the 3rd International Conference on Application and Theory of Automation in Command and Control Systems, Naples, Italy. https://doi.org/10.1145/2494493.2494515

Jung, J., D'Souza, S. N., Johnson, M. A., Ishihara, A. K., Modi, H. C., Nikaido, B., \& Hasseeb, H. (2016). Applying required navigation performance concept for traffic management of small unmanned aircraft systems. Retrieved from https://utm.arc.nasa.gov/docs/Jung_ICAS_2016.pdf 
Kadhiresan, A. R., \& Duffy, M. J. (2019). Conceptual design and mission analysis for eVTOL urban air mobility flight vehicle configurations. AIAA Journal Papers. https://doi.org/10.2514/6.2019-2873

Kopardekar, P., Rios, J., Prevot, T., Johnson, M., Jung, J., \& Robinson, J. E. (2016). Unmanned Aircraft System Traffic Management (UTM) Concept of Operations. $16^{\text {th }}$ AIAA Aviation Technology, Integration, and Operations Conference, Washington, DC. https://doi.org/10.2514/6.2016-3292

Lascara, B., Lacher, A., DeGarmo, M., Maroney, D., Niles, R., \& Vempati, L. (2019, June). Urban air mobility airspace integration concepts. Operational Concepts and Exploration Approaches. The MITRE Corporation. Retrieved from https://www.mitre.org/sites/default/files/publications/pr-19-00667-9-urban-air-mobilityairspace-integration.pdf

Moore, M. D. (2010). Aviation Frontiers - On-Demand Aircraft. 10th AIAA Aviation Technology, Integration, and Operations Conference, Fort Worth, TX. https://doi.org/10.2514/6.2010-9343

Mueller, E., Kopardekar, P., \& Goodrich, K. H. (2017). Enabling airspace integration for highdensity on-demand mobility operations. NASA Center for AeroSpace Information (CASI). Conference Proceedings, Hampton, VA. https://doi.org/10.2514/6.2017-3086

National Aeronautics and Space Administration (NASA). (2018). Urban air mobility (UAM) market study. Retrieved from https://ntrs.nasa.gov/archive/nasa/casi.ntrs.nasa.gov/20190026762.pdf 
National Aeronautics and Space Administration (NASA). (2019). UAM coordination and assessment team (UCAT). NASA UAM for ENRI Technical Interchange Meeting. Retrieved from https://ntrs.nasa.gov/archive/nasa/casi.ntrs.nasa.gov/20190025373.pdf

Reiche, C., McGillen, C., Siegel, J., \& Brody, F. (2019). Are we ready to weather urban air mobility (UAM)? Integrated Communications, Navigation and Surveillance Conference (ICNS), Herndon, VA. https://doi.org/10.1109/ICNSURV.2019.8735297

Thacker, M. (2018). Urban air mobility: The way forward. Retrieved from https://docs.house.gov/meetings/SY/SY00/20180724/108586/HHRG-115-SY00-WstateThackerM-20180724.pdf

Thipphavong, D. P., Apaza, R. D., Barmore, B. E., Battiste, V., Belcastro, C. M., Burian, B. K., . . . Verma, S. A. (2018). Urban air mobility airspace integration concepts and considerations. AIAA Aviation Forum, 2018 Aviation, Technology, Integration, and Operations Conference, Atlanta, GA. https://doi.org/10.2514/6.2018-3676

Vascik, P. D., \& Hansman, R. J. (2018). Scaling constraints for urban air mobility operations: Air traffic control, ground infrastructure, and noise. AIAA Aviation Forum, 2018 Aviation, Technology, Integration, and Operations Conference, Atlanta, GA. https://doi.org/10.2514/6.2018-3849

Vascik, P. D., Hansman, R. J., \& Dunn, N. S. (2018). Analysis of urban air mobility operational constraints. Journal of Air Transportation, 26(4), pp. 133-146. https://doi.org/10.2514/1.D0120

Verma, S., Keeler, J., Edwards, T. E., \& Dulchinos, V. (2019). Exploration of near-term potential routes and procedures for urban air mobility. AIAA Journal Papers. https://doi.org/10.2514/6.2019-3624 